\title{
A case report: incomplete Kawasaki disease in a hypogammaglobulinemic child
}

\author{
Burçin Şanlıdă̆, M.D. ${ }^{a}$, Ceyhun Dalkan, Assoc. Prof., M.D. ${ }^{a}$ and Nerin Bahçeciler, Prof., M.D. ${ }^{a}$
}

\begin{abstract}
Kawasaki Disease (KD) is a systemic autoimmune vasculitis that affects small and medium sized vessels. Main complication of Kawasaki Disease is coronary artery aneurism, which has higher riskin case of delayed diagnosis and treatment. Although, complete and incomplete KD cases in different types of immune deficiency diseases have been presented up to date, clinical course of KD in patients with hypogammaglobulinemia (HG) has not been reported. Herein, a case diagnosed as incomplete KD in a child with transient HG of infancy has been reported. Previously reported cases with KD and immunedeficiency have also been summarized.

Recurrent infections in case of immunedeficiency may mask KD disease resulting in delay in diagnosis and increased risk of complication. KD should be kept in mind in immunedeficient patients in case of prolonged fever.

Key words: Kawasaki disease, hypogammaglobulinemia, immune deficiency.
\end{abstract}

http:/ / dx.doi.org/10.5546/ aap.2018.eng.e322

To cite: Şanlıdağ B, Dalkan C, Bahçeciler N. A case report: incomplete Kawasaki disease in a hypogammaglobulinemic child. Arch Argent Pediatr 2018;116(2):e322-e324.

\section{INTRODUCTION}

Kawasaki disease (KD) known as mucocutaneus lymph node syndrome, is a systemic, autoimmune vasculitis involving small and medium sized vessels which mainly affects children. ${ }^{1} \mathrm{KD}$ is the leading cause of acquired

a. Department of Pediatrics, Near East University, Nicosia, Cyprus.

E-mail address:

Burçin Şanlıdă̆, M.D.: burcinsanlidag@yahoo.com

Funding: None.

Conflict of interest: None.

Received: 7-13-2017

Accepted: 10-9-2017 heart disease in children in which coronary artery aneurism develops in about $25-30 \%$ of untreated patients. ${ }^{2}$ The use of intravenous immunglobuline (IVIG), aspirin and warfarin within the first 10 days of the onset of symptoms reduce the risk of coronary artery complications. ${ }^{3}$ Diagnosis of $\mathrm{KD}$ is based on the criteria defined by American Heart Assosiation. Some cases are diagnosed as incomplete KD if not fulfill criteria. Although clinically less prominent, development of vascular complications is also possible in incomplete KD. ${ }^{4,5}$ Some reports have indicated that incomplete Kawasaki itself is associated with coronary artery aneurism development because of delay in treatment. ${ }^{6,7}$

Pathogenesis is not clear in KD; immune response differs in acute and later phase of the disease. ${ }^{8-10}$ Possibly, hyperactivation and dysfunction of the immune system triggered by an unknown ethiological agent might cause a subtle clinic resulting in incomplete KD. The course of this more subtle clinical presentation in patients with additional hypogammaglobulinemia (HG) is not defined yet.

$\mathrm{KD}$ in patients with immune deficiency disorders has been reported rarely in isolated cases with Wiscott-Aldrich syndrome, hyperimmunoglobulin E syndrome, Chronic granulomatosis disease (CGD) and selective Ig A deficiency. ${ }^{11-14}$ The impact of HG on the onset and course of KD has not been reported yet.

A four years old boy with HG who developed incomplete KD has been presented. Aim of the presented case is to emphasize the importance of early diagnosis of KD in a patient with $\mathrm{HG}$ in order to protect against coronary artery aneurism.

\section{CASE}

A four year old boy who had recurrent tonsillitis and bronchopneumonia was diagnosed previously (6 months ago) as HG. He was admitted with complaints of high fever (axillary $40{ }^{\circ} \mathrm{C}$ ) which started the previous day. Physical examination was normal except for strawberry tongue.

Initial levels of serum total Ig G and Ig A were below 2 SD levels appropriate for age. 
Lymphocyte subset analysis was normal. Antibody responses to streptococcus pneumonia and tetanus, and isohemagglutinin levels were normal. He had been under follow up with trimetoprim sulphamethaxazole prophylaxis and recurent infections were under control throughout the 6 months treatment period. Based on his immunological evaluation he was diagnosed as transient hypogammaglobulinemia of infancy.

At the final attendance laboratory evaluation revealed WBC: $24.600 \mu \mathrm{L}, 76 \%$ neutrophile predominance. Platelet count was $808.000 \mu \mathrm{L}$, sedimentation rate and $\mathrm{C}$-reactive protein were 20 and $12.2(0-0.5)$ respectively. Serology for Ebtein Barr Virus, Cytomegalovirus, Adenovirus were negative and throat, urine, blood cultures were obtained which resulted as negative. A broad spectrum antibiotic treatment was initiated. In the follow up conjunctivitis appeared on the fourth day, fever subsided on the $6^{\text {th }}$ day and on the $10^{\text {th }}$ day membranous desquamation of fingertips of hands and feet were observed. Fever persisted for more than 5 days. And he had fullfiled 3 of 5 criteria for KD established by American Heart Association; ${ }^{6,7}$ he was diagnosed as incomplete KD. No aneurysm was detected on echocardiography (ECHO). IVIG treatment with a dosage of $2 \mathrm{~g} / \mathrm{kg}$ and aspirin $30 \mathrm{mg} / \mathrm{kg} /$ day were initiated. Aspirin was diminised and stopped after resolution of symptoms and normalisation of acute phase reactants. ECHO was repeated on the $15^{\text {th }}$ day with normal results.

\section{DISCUSSION}

Herein; a 4 year old boy with transient HG of infancy who developed incomplete $\mathrm{KD}$ is presented to emphasize the difficulty in diagnosis of KD in immune deficiency situations. Previously; KD cases in various immune deficiency diseases had been reported including; Wiscot Aldrich syndrome, hyperımmunoglobulin E syndrome, CGD and selective Ig A deficiency. ${ }^{11-14}$ Among those cases, a patient with CGD had presented with incomplete $\mathrm{KD}$, who had been treated with IVIG on the $18^{\text {th }}$ day and unfortunately developed coronary artery disease. The CGD case had been treated as suppurative cervical lypmhadenitis initially. In the patient with selective Ig A deficiency diagnosis of KD had been established on the $5^{\text {th }}$ day and was treated with aspirin, urinastatin and steroid pulse therapy instead of IVIG. No coronary artery aneurism developed. The case with Wiscott-Aldrich syndrome was diagnosed as complete $\mathrm{KD}$ at 6 months of age with transient normalisation of platelet count during disease course. This patient had been treated with IVIG with no complications. Coronary artery aneurism formed only in the case of CGD among all those previously reported cases, which might be due to delay in IVIG treatment. ${ }^{11-14}$

Pathogenesis of KD has not been fully understood. It is hypothesized that; a possible infectious agent causes pathogenic substances to be produced, spread and bound to endothelial cells of both small and medium sized blood vesssels. Immmune system has been activated for control. Firstly; non-specific T cells and antibodies are hyperactivated, then cytokine production happens and ends with further endothelial injury. By the resolution of the inflammation involving vessels, specific $T$ cells and antibodies are released against pathogenic proteins and repair process begins. ${ }^{8}$ Both the activation and the dysfunction of the immune system are involved in the acute phase of the disease. ${ }^{9}$

Incomplete $\mathrm{KD}$ is also associated with the development of coronary artery aneurism. ${ }^{8,9}$ As the diagnosis of incomplete KD is difficult to establish, vascular damage may progress before onset of the treatment. Coronary artery aneurism develops in 25-30\% of untreated cases. Most important factor to protect from complication is early diagnosis and early initiation of IVIG treatment within 10 days of symptom onset. ${ }^{3}$

Rowley et al postulated that the ethiological agent in KD enters through the respiratory tract stimulating an early Ig A and Ig M immune response, with an IgG response developing later in the disease course. ${ }^{10}$ The underlying cause of incomplete KD in the presented case may be the incomplete immune response due to hypogammaglobulinemia. Hypogammaglobulinemia may result in less antibody response involved in pathogenesis of $\mathrm{KD}$ which may result in an incomplete clinical presentation and end up with delay in diagnosis and therefore treatment.

In a recent study elevated $B$ cells and $C 3$ levels were detected in $K D$, that may indicate the predominance of humoral immune response in KD. ${ }^{15}$ The percentage of CD19+ cells was markedly elevated in complete KD; showing that $\mathrm{B}$ cell-mediated immune reactions appear to be the primary underlying mechanism. Low expression of $\mathrm{T}$ cells and high expression of $\mathrm{B}$ cells might be associated with complete KD 
while incomplete KD may be characterised by a higher T cell level. ${ }^{15}$ In addition, patients who were sensitive to IVIG treatment were found to have decreased CD 19+ cells. ${ }^{15}$ This data may postulate that decreased levels of antibodies in our case might exert a sensitivity to IVIG treatment, thereby protection from coronary artery aneurism.

In conclusion; in children with $\mathrm{HG}, \mathrm{KD}$ should be included in differential diagnosis of high grade prolonged fever, as treatment of KD prompts immediate IVIG treatment in order to prevent coronary artery disease. In addition low Ig G levels may result in a more subtle immune response, clinical presentation and IVIG sensitivity.

\section{REFERENCES}

1. Yim D, Curtis N, Cheung M, et al. An update on Kawasaki disease II: clinical features, diagnosis, treatment and outcomes. J Paediatr Child Health 2013;49(8):614-23.

2. Uehara R1, Belay ED. Epidemiology of Kawasaki disease in Asia, Europe, and the United States. J Epidemiol 2012;22(2):79-85.

3. Soriano-Ramos M, Martínez-Del Val E, Negreira Cepeda $\mathrm{S}$, et al. Risk of coronary artery involvement in Kawasaki disease. Arch Argent Pediatr 2016;114(2):107-13.

4. Newburger JW, Takahashi M, Gerber MA, et al. Diagnosis, treatment, and long-term management of Kawasakidisease: a statement for health professionals from the Committee on Rheumatic Fever, Endocarditis and Kawasaki Disease,
Council on Cardiovascular Disease in the Young, American Heart Association. Circulation 2004;110(17):2747-71.

5. Belay ED, Maddox RA, Holman RC, et al. Kawasaki syndrome and risk factors for coronary artery abnormalities: United States, 1994-2003. Pediatr Infect Dis J 2006;25(3):245-9.

6. Sonobe T, Kiyosawa N, Tsuchiya K, et al. Prevalence of coronary artery abnormality in incomplete Kawasaki disease. Pediatr Int 2007;49(4):421-6.

7. Yeo Y, Kim T, Ha K, et al. Incomplete Kawasaki disease in patients younger than 1 year of age: a possible inherent risk factor. Eur J Pediatr 2009;168(2):157-62.

8. Lee KY, Rhim JW, Kang JH. Kawasaki disease: laboratory findings and an immunopathogenesis on the premise of a "protein homeostasis system". Yonsei Med J 2012;53(2):262-75.

9. Yeung RS. Kawasaki disease: update on pathogenesis. Curr Opin Rheumatol 2010;22(5):551-60.

10. Rowley AH, Miura M. Immunoglobulin A deficiency and Kawasaki disease. Pediatr Int 2010;52(2):330-1.

11. Muneuchi J, Ishimura $\mathrm{M}$, Takada $\mathrm{H}$, et al. Incomplete Kawasaki disease in a patient with chronic granulomatous disease. Pediatr Int 2010;52(3):e134-6.

12. Kawakami C, Miyake M, Tamai H. Kawasaki disease in a patient with Wiskott-Aldrich syndrome: an increase in the platelet count. Int J Hematol 2003;77(2):199-200.

13. Kimata H. High-dose intravenous gamma-globulin treatment for hyperimmunoglobulinemia E syndrome. J Allergy Clin Immunol 1995;95(3):771-4.

14. Nishikawa T, Nomura $\mathrm{Y}$, Kono $\mathrm{Y}$, et al. Selective IgA deficiency complicated by Kawasaki syndrome. Pediatr Int 2008;50(6):816-8.

15. Ding Y, Li G, Xiong LJ, et al. Profiles of responses of immunological factors todifferent subtypes of Kawasaki disease. BMC Musculoskelet Disord 2015;23;16:315. 